\title{
Characterization of Trypanosoma brucei isolated from lymph nodes of rats
}

\author{
M. TANNER, L. JENNI, H. HECKER and R. BRUN \\ Swiss Tropical Institute, Socinstrasse 57, CH-4051 Basel, Switzerland
}

(Accepted 2 July 1979)

\begin{abstract}
S UMMARY
Forms of Trypanosoma brucei isolated from lymph nodes of cyclically or intravenously infected rats, at different stages of infection, were morphologically compared with the corresponding bloodstream forms. Unlike the pleomorphic bloodstream trypanosome population, the lymph node trypanosome population was essentially monomorphic and resembled the slender forms of the bloodstream. Values for the morphometric parameters indicated a position between the slender and the stumpy forms. The number of trypanosomes $/ \mathrm{ml}$ of blood showed the well-known periodic fluctuations while the number of trypanosomes/g of lymph node remained fairly constant. Serology revealed that lymph node trypanosome populations differed antigenically from the trypanosome populations isolated simultaneously from the bloodstream.
\end{abstract}

\section{INTRODUCTION}

Trypanosomes of the Trypanosoma brucei sub-group multiply not only in the vascular system but also extravascularly (Losos \& Tkede, 1972). In particular they seem to have a predilection for the lymphoid system and for connective tissue (Schuberg \& Boeing, 1913; Goodwin, 1970). Amastigote tissue forms and foci of development have been described in the internal organs of vertebrate hosts (Soltys, Woo \& Gillick, 1969). Ormerod \& Venkatesan (1971) have speculated that amastigote forms in the choroid plexus of the $T$. brucei-infected vertebrate host could be of great importance for the bloodstream trypanosome population. In their view, the amastigotes are the forms which give rise to slender, dividing trypomastigotes circulating in the blood. In addition, Ssenyonga \& Adam (1975) obtained evidence that monomorphic, slender-like forms of $T$. brucei are flushed from the lymphatic system into the bloodstream.

The importance of tissue foci of $T$. brucei sub-group trypanosomes has not often been assessed in relation to the antigenic nature of these populations. Seed \& Effron (1973) have shown that trypanosomes from different parts of rodent brains infected with $T$. gambiense differ antigenically from trypanosomes isolated simultaneously from the blood. Balber (1972) has suggested that slender forms invade tissue spaces, where they undergo antigenic variation.

In the present study, we compare morphologically and serologically the trypanosomes found in the lymph nodes of $T$. brucei-infected rats at different stages of infection with simultaneously isolated bloodstream trypanosomes. 


\section{MATERIALS AND METHODS}

Trypanosoma brucei stocks

Stabilate STIB 348T is a clone derived from a single metacyclic form of stabilate STIB 348, which is itself a cloned derivative of STIB 246. STIB 246 was originally isolated from a hartebeest (Alcelaphus buselaphus) in the Serengeti (Tanzania) in 1971 and cryopreserved after 1 rat passage (Geigy, Jenni, Kauffmann, Onyango \& Weiss, 1975). Stabilate STIB $366 \mathrm{~A}$ is a clone of primary isolate Serengeti/66/ SVRP/42 (Baker, 1979) which we received from the Molteno Institute, Cambridge.

\section{Animals}

SIV white rats (females, 200-230 g) and ICR white mice (females, 20-28 g) were used (Tierzuchtinstitut, Universität Zürich, Switzerland). Pupae of Glossina $m$. morsitans were obtained from Dr A. M. Jordan, Langford, Bristol. The pupae were maintained and the teneral flies infected with STIB $348 \mathrm{~T}$ as described by Jenni (1977).

\section{Infection}

Stabilate STIB 348T: each rat was infected by a single infectious bite.

Stabilate STIB 366A: after thawing the stabilates, the organisms were diluted with ESG-buffer (ethylenediaminetetra-acetic acid saline-glucose, $\mathrm{pH} 7 \cdot 4$, Walker (1970)) and $10^{6}$ motile trypanosomes were injected intravenously (tail vein) into each rat.

\section{Bloodstream trypanosomes $(B F)$}

Tail blood was examined daily for trypanosomes by the haematocrit centrifuge technique (HCT) or by direct wet preparation. Counts were made in a Neubauer haemocytometer. Blood smears were prepared daily, fixed with methanol and Giemsa-stained. At least 250 trypanosomes were examined and classified as slender, dividing, intermediate or stumpy forms.

\section{Lymph node trypanosomes $(L F)$}

Rats (6/group) were killed at the 1st peak, the remission or the 2nd peak of parasitaemia and the following lymph nodes were removed and kept in ice-cold phosphate-buffered saline-glucose (PSG) 6:4, pH 8.0: axillary-, inguinal-, pharyngeal-, lumbar-, mesenteric- and portal vein-lymph nodes. The lymph nodes were weighed, pooled and triturated in ice-cold PSG with a loose-fitting teflon pestle. The trypanosomes in the resulting cell suspension were counted and the corresponding number of trypanosomes/g of lymph node was calculated. Thin films of the suspension were prepared and examined as described above.

\section{Quantitative morphological investigation of $L F$}

Cyclically infected rats were killed at the 2nd peak of parasitaemia (10-15 days post-infection). Trypanosomes were separated from the lymph node cells by centrifugation for $20 \mathrm{~min}$ at $60 \mathrm{~g}$ and $4^{\circ} \mathrm{C}$. The trypanosomes recovered from the supernatant fluid were washed 4 times in PSG and prepared for light and electron microscopy. 


\section{Light microscopy}

Thin smears were prepared and the mean absolute nuclear volume $(V n u)$ of the trypanosomes was evaluated (Hecker, Burri, Steiger \& Geigy, 1972).

\section{Electron microscopy}

Processing of entire infected lymph nodes as well as pellets of separated LF was carried out by standardized methods (Hecker et al. 1972). The sampling for morphometry and the test systems used were essentially the same as previously described (Hecker et al. 1972; Hecker, Burri \& Boehringer, 1973). The following morphometric parameters were investigated and their mean values \pm standard errors (s.w.) were calculated: absolute volume of nucleus ( $V n u)$, absolute volume of cytoplasm without flagellum ( $V c y)$, nuclear/cytoplasmic ratio $(n u / c y)$, volume density of mitochondrion $\left(V_{\nabla m i}\right)$, volume density of glycosomes $\left(V_{\nabla g l}\right)$, surface density of mitochondrial outer membrane $\left(S_{V_{m i o}}\right)$, surface density of mitochondrial inner membrane $\left(S_{V m i i}\right)$. Groups of results were obtained from each of 2 infected rats and compared statistically (Student's $t$-test, significance limit $2 P<0.05$ ).

\section{Serology}

BF and LF were separated from blood cells or lymphocytes by centrifugation in the cold as described above. The trypanosomes were washed 4 times, fixed in $1 \%$ formalin and prepared for the indirect fluorescent-antibody test (IFAT) as described by Nantulya \& Doyle (1977).

Sera were obtained from the infected rats at the same time as the rats were killed for the isolation of BF and LF. Inactivated sera were tested by IF AT against procyclic culture forms of the homologous trypanosome stock (grown in SDM-77, Brun \& Jenni (1977)) to detect antibodies against common antigens. As all sera no longer reacted with procyclic culture forms at a dilution of $1: 320,2$-fold dilutions starting at 1:320 were used to test for variable antigen types (VAT). Drops of serum dilutions (phosphate-buffered saline (PBS), $\mathrm{pH} 7 \cdot 3$, as diluent) were allowed to react with fixed trypanosome samples at $37{ }^{\circ} \mathrm{C}$ for 25 min. The slides were washed 3 times in PBS for $10 \mathrm{~min}$. The trypanosomes were subsequently incubated in a 1:80 dilution of a fluorescein-isothiocyanate-conjugated rabbit anti-rat (heavy and light chains) IgG (Cappel Laboratories, Cochranville, Pa., USA) at $37^{\circ} \mathrm{C}$ for $25 \mathrm{~min}$. The slides were again washed as above and mounted in phosphate-buffered glycerol. Control tests included incubation in normal rat serum or incubation in conjugate alone. At least 200 trypanosomes were examined under a Zeiss fluorescence phase-contrast microscope and the proportion of positively and negatively stained forms was determined.

\section{RESULTS}

Groups of 6 intravenously infected rats were killed at the 1st peak, the remission and the 2nd peak of parasitaemia. As demonstrated in Fig. 1, the number of trypanosomes $/ \mathrm{ml}$ of blood showed the well-known periodic fluctuation of a bloodstream population, while the number of $\mathrm{LF} / \mathrm{g}$ of lymph node remained fairly 


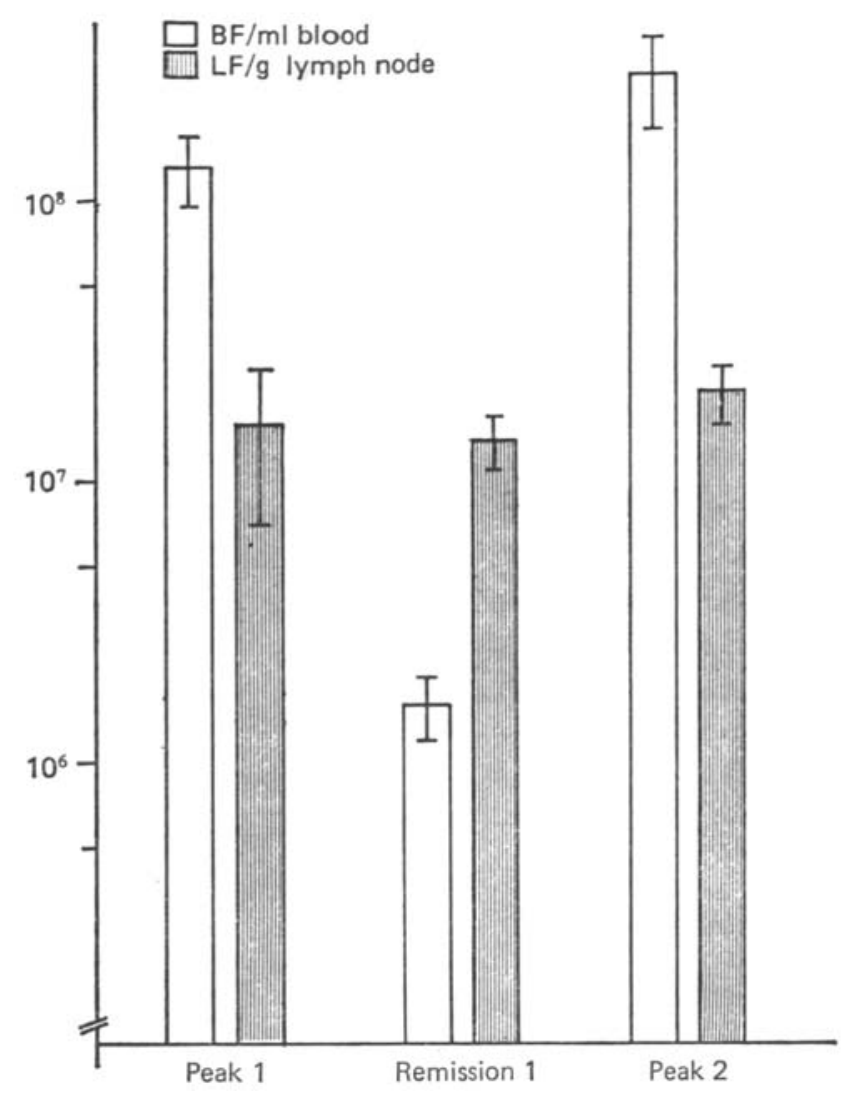

Fig. 1. Number of trypanosomes in $1 \mathrm{ml}$ of blood (BF) and number of trypanosomes in $1 \mathrm{~g}$ of lymph node (LF) simultaneously isolated from rats intravenously infected with $10^{6}$ Trypanosoma brucei (stabilate STIB 366A) at the 1st peak, the remission and the 2nd peak of parasitaemia. Each column represents the arithmetic mean \pm s.t. of 6 rats.

constant during infection (i.e. until the 2nd peak of parasitaemia which was reached between days 11 and 15 post-infection).

Fig. 2 shows that the percentage of dividing forms in the lymph node population was usually higher than in the corresponding bloodstream population in cyclically as well as in intravenously infected rats. Two and three days after intravenous infection, no parasites were detectable by HCT in the blood of 2 rats (triplicate HCT of tail blood, heart blood and hepatic vein blood), although the lymph node trypanosome populations were already established and showed a remarkably high dividing rate (Fig. $2 \mathrm{~B}$ ).

LF were infective (i.p. as well as i.v.) for mice and rats at all the stages of infection. The parasitaemia resulting from injection of LF was not distinguishable in its course and pleomorphism from a parasitaemia caused by the same number of $\mathrm{BF}$. It was also possible to obtain clones from lymph node populations. LF could not be adapted to a vector-form culture although the semi-defined medium used, SDM-77 (Brun \& Jenni, 1977), enabled direct adaptation of intermediate and stumpy BF of the same stock into a vector-form culture. 


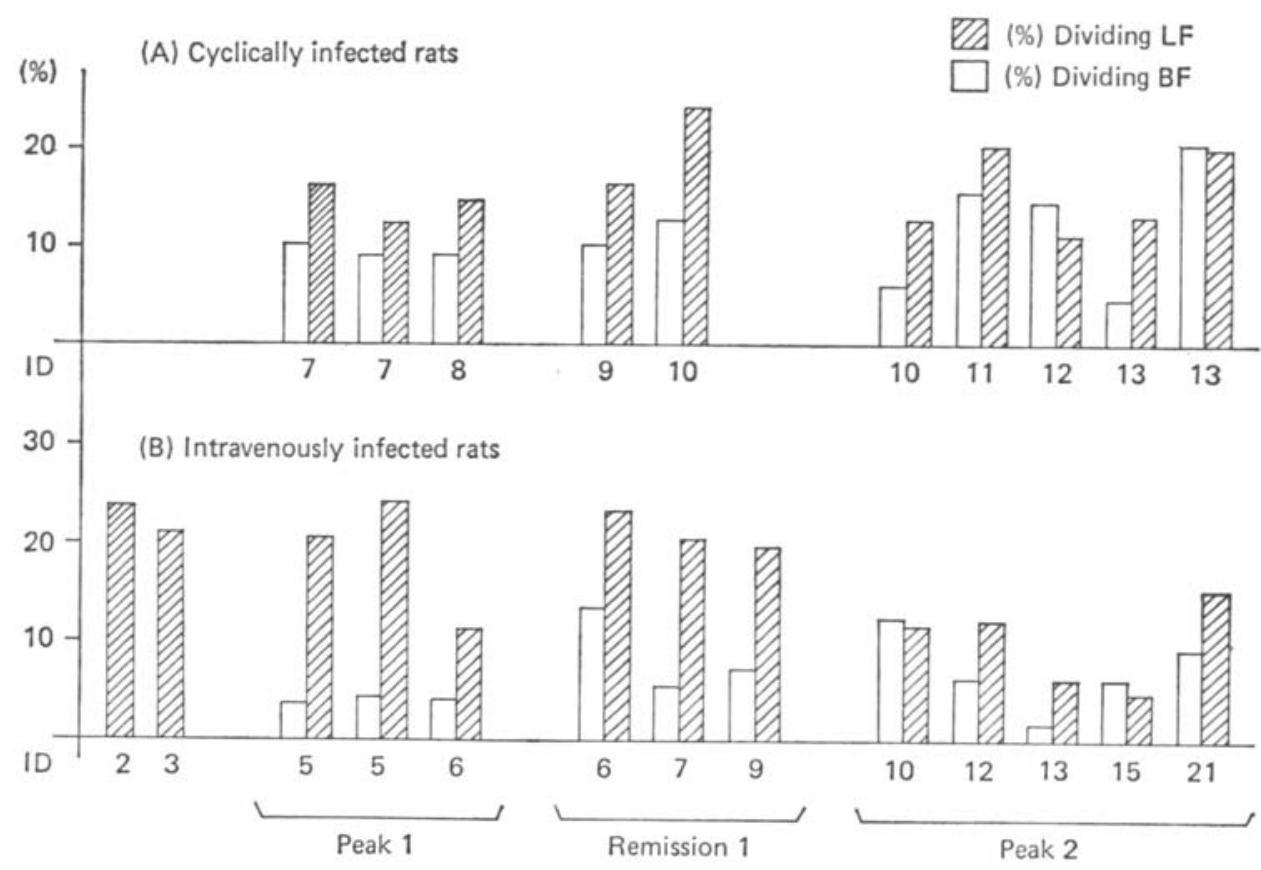

Fig. 2. Proportion of dividing forms in the blood and Iymph nodes of rats infected with Trypanosoma brucei (stabilate STIB 366A for intravenous infection, stabilate STIB 348T for cyclical infection). Each double column shows the data of a single rat. Rats were killed at the 1st peak, the remission or the 2nd peak of parasitaemia. $\mathrm{BF}$, trypanosomes isolated from the blood; LF, trypanosomes isolated from the Iymph nodes; ID, days after infection.

LF had an intermediate-slender appearance (PI. 1 A). Stumpy forms could only be detected sporadically $(<2 \%$ ) among trypanosomes isolated from lymph nodes at different stages of parasitaemia (peaks and remission). At the same time the proportion of stumpy forms circulating in the blood varied between $11.8 \pm 2.0 \%$ and $21.2 \pm 4.9 \%$ (means \pm S.E., $n=16$ ). The lymph node trypanosome populations appeared fairly monomorphic during all the stages of infection. In contrast, the bloodstream trypanosome populations showed the well-known pleomorphism. Electron microscopy revealed that the LF occurred predominantly intercellularly in the lymph node tissue (Pl. 1C). LF were found in all regions of the lymph nodes. No predilection for a particular site could be noted. LF also had a surface coat (Pl. $1 \mathrm{~B}$, inset) which was similar in thickness and electron density to the BF. Morphometric data for LF are presented in Table 1. The quantitative morphology of LF was compared with previously published values for slender and stumpy BF of rats infected with other T. brucei stocks (Boehringer \& Hecker, 1974, 1975, values included in Table 1). It could thus be demonstrated that the values of most of the parameters for LF lie between those for slender and those for stumpy forms. This is especially clear in the case of the mitochondrial values. The results for $\nabla n u$, $n u / c y, S_{V m i i}$ and $V_{V \rho l}$ obtained from 2 different rats were significantly different.

The reaction pattern of 3 cumulative infection sera harvested from 3 rats at different states of infection revealed that the lymph node trypanosome population contained a different set of antigenic populations than the corresponding blood- 
Table 1. Trypanosoma brucei stabilate STIB 348T, trypanosomes isolated from lymph nodes $(L F)$ of cyclically infected rats

(Mean \pm s.e. of parameter results (for abbreviations see Materials and Methods section).)

\begin{tabular}{|c|c|c|c|c|c|c|c|}
\hline & $\begin{array}{l}V n u \\
\left(\mu \mathrm{m}^{3}\right)\end{array}$ & $n u / c y$ & $\begin{array}{c}V c y \\
\left(\mu \mathrm{m}^{3}\right)\end{array}$ & $V_{V_{m} s}$ & $S_{V_{m+1}}$ & $S_{V_{m i t}}$ & $V_{V g l}$ \\
\hline $\begin{array}{l}\text { LF STIB 348T } \\
\text { Rat 38 }\end{array}$ & $\begin{array}{r}4 \cdot 12 \\
\pm 0 \cdot 18\end{array}$ & $\begin{array}{r}0.170 \\
\pm 0.015\end{array}$ & $24 \cdot 2$ & $\begin{array}{r}0.059 \\
+0.008\end{array}$ & $\begin{array}{r}0.968 \\
+0.099\end{array}$ & $\begin{array}{r}1.065 \\
\pm 0.128\end{array}$ & $\begin{array}{r}0.062 \\
\pm 0.004\end{array}$ \\
\hline $\begin{array}{l}\text { LF STIB 348T } \\
\text { Rat 40 }\end{array}$ & $\begin{array}{r}3.04 \\
\pm 0.16\end{array}$ & $\begin{array}{r}0.131 \\
+0.015\end{array}$ & $23 \cdot 2$ & $\begin{array}{r}0.068 \\
+0.005\end{array}$ & $\begin{array}{r}1.154 \\
+0.060\end{array}$ & $\begin{array}{r}1.400 \\
\pm 0.094\end{array}$ & $\begin{array}{r}0.077 \\
\pm 0.005\end{array}$ \\
\hline $\begin{array}{l}\text { SL STIB 348T } \\
\text { Rat } 38\end{array}$ & $\begin{array}{r}1.71 \\
+0.10\end{array}$ & N.D. & N.D. & N.D. & N.D. & N.D. & N.D. \\
\hline $\begin{array}{l}\text { SL STIB 348T } \\
\text { Rat 40 }\end{array}$ & $\begin{array}{r}1 \cdot 62 \\
\pm 0 \cdot 16\end{array}$ & N.D. & N.D. & N.D. & N.D. & N.D. & N.I \\
\hline $\begin{array}{l}\text { SL } \\
\text { STIB 338* } \\
\text { STIB 33** }\end{array}$ & $\begin{array}{l}2 \cdot 0 \\
3 \cdot 0\end{array}$ & $\begin{array}{l}0 \cdot 110 \\
0 \cdot 106\end{array}$ & $\begin{array}{l}14 \cdot 1 \\
20 \cdot 0\end{array}$ & $\begin{array}{l}0.092 \\
0.045\end{array}$ & $\begin{array}{l}1.03 \\
0.80\end{array}$ & $\begin{array}{l}1.50 \\
0.92\end{array}$ & $\begin{array}{l}0.080 \\
0.084\end{array}$ \\
\hline SL STIB 65* & $1 \cdot 6$ & 0.126 & $9 \cdot 1$ & 0.033 & 0.67 & 0.60 & 0.090 \\
\hline $\begin{array}{l}\text { SS STIB 348T } \\
\text { Rat } 38\end{array}$ & $\begin{array}{r}6.83 \\
\pm 0.38\end{array}$ & N.D. & N.D. & N.D. & N.D. & N.D. & N.D. \\
\hline $\begin{array}{l}\text { SS STIB 348T } \\
\text { Rat 40 }\end{array}$ & $\begin{array}{r}5.69 \\
\pm 0.16\end{array}$ & N.D. & N.D. & N.D. & N.D. & N.D. & N.I \\
\hline SS STLB 338* & $5 \cdot 5$ & $0 \cdot 110$ & $39 \cdot 7$ & 0.146 & $1 \cdot 6$ & $2 \cdot 79$ & $\begin{array}{l}0.070 \\
0.076\end{array}$ \\
\hline STIB 33* & $7 \cdot 4$ & & & & 1.39 & $2 \cdot 47$ & $\begin{array}{l}0.076 \\
0.078\end{array}$ \\
\hline SS STIB $65^{*}$ & $3 \cdot 7$ & 0.124 & $22 \cdot 4$ & 0.147 & 1.37 & $3 \cdot 13$ & 0.078 \\
\hline
\end{tabular}

N.D., not done.

* Extrapolated mean values for slender forms (SL) and short stumpy forms (SS) from Boehringer \& Hecker (1974).

Table 2. Trypanosoma brucei stabilate STIB 366A: immunofluorescent staining of trypanosomes isolated from the blood $(B F)$ and lymph nodes $(L F)$ at different days after intravenous infection (ID) with the corresponding infection sera

(Numbers indicate percentage of positively stained parasites. Rem., remission of parasitaemia ; NRS, normal rat serum; CF, culture forms;,$-<1 \%$.)

Rat sera $(1: 320)$ isolated at

\begin{tabular}{lccccc}
\multicolumn{1}{c}{$\begin{array}{c}\text { Peak 1 } \\
\text { Antigen }\end{array}$} & $\begin{array}{c}\text { Rem. 1 } \\
\text { (ID 6) }\end{array}$ & $\begin{array}{c}\text { Peak 2 } \\
\text { (ID 8) }\end{array}$ & $\begin{array}{c}\text { NRS } \\
\text { (ID 12) }\end{array}$ & $\begin{array}{c}\text { Conjugate } \\
1: 320\end{array}$ \\
BF peak 1 & $>95$ & 90 & $>95$ & - & - \\
LF peak 1 & - & - & 80 & - & - \\
BF rem. 1 & 60 & $>95$ & $>95$ & - & - \\
LF rem. 1 & - & 30 & 90 & - & - \\
BF peak 2 & - & 75 & 80 & - & - \\
LF peak 2 & - & - & 50 & - & - \\
Stabilate STIB 366A & $>95$ & $>95$ & $>95$ & - & - \\
Procyclic CF STIB 366A & - & - & - & -
\end{tabular}


Table 3. Trypanosoma brucei stabilate STIB 348T : immunofluorescent staining of trypanosomes isolated from the blood $(B F)$ and lymph nodes $(L F)$ at different days after cyclical infection $(I D)$ with the corresponding infection sera

(Numbers indicate percentage of positively stained parasites. Rem., remission of parasitaemia ; NRS, normal rat serum; CF, culture forms ;,$<1 \%$.)

\begin{tabular}{lccccc}
\multicolumn{7}{c}{ Rat sera $(1: 320)$ isolated at } \\
Antigen & $\begin{array}{c}\text { Peak 1 } \\
\text { (ID 7) }\end{array}$ & $\begin{array}{c}\text { Rem. 1 } \\
(\text { ID 10) }\end{array}$ & $\begin{array}{c}\text { Peak 2 } \\
\text { (ID 11) }\end{array}$ & $\begin{array}{c}\text { NRS } \\
1: 320\end{array}$ & $\begin{array}{c}\text { Conjugate } \\
1: 80\end{array}$ \\
BF peak 1 & 40 & 30 & 40 & - & - \\
LF peak 1 & - & - & 25 & - & - \\
BF rem. 1 & 80 & 90 & 10 & - & - \\
LF rem. 1 & - & 5 & 80 & - & - \\
BF peak 2 & - & 10 & 25 & - & - \\
LF peak 2 & - & - & - & - & -
\end{tabular}

stream trypanosome population from the same animal (Table 2). IFAT results with sera and antigens from cyclically infected rats (Table 3) were similar to those from intravenously infected rats. Again, the lymph node trypanosome populations seemed to differ antigenically from bloodstream trypanosome populations simultaneously isolated from the same animal.

\section{DISC USSION}

The intravenous injection of trypanosomes demonstrated that an extravascular trypanosome population was well established in the lymph nodes as early as the 1st peak of parasitaemia. The careful studies of Schuberg \& Boeing (1913) revealed that tissue spaces of lymphoid organs are a favoured site for $T$. brucei to multiply. The trypanosomes isolated from lymph nodes (LF) resembled bloodstream slender forms as was shown by light microscopy. The failure to adapt them into a vectorform culture also supports the slender-like nature of LF, as it is known that the trypanosomes need the partially activated mitochondrion characteristic of intermediate and stumpy forms for the transformation into procyclic culture forms (Brown, Evans \& Vickerman, 1973). In addition, the LF were compared morphometrically with slender and stumpy forms derived from other trypanosome stocks (Boehringer \& Hecker, 1974, 1975). The position of the LF between slender and stumpy forms was thereby confirmed.

It is interesting to note that, in the same animal, a pleomorphic and a monomorphic parasite population grow simultaneously. It is not clear if only slender forms invade tissue spaces (as assumed by Balber, 1972) or if stumpy and intermediate-stumpy forms are rapidly destroyed in the lymph nodes. Ssenyonga \& Adam (1975) suggested that a monomorphic trypanosome population is constantly flushed from lymph into blood. This trypanosome population was very similar to our lymph node trypanosome population in its morphology as well as in its dividing rate. 
Using cumulativei nfection sera and testing them at a dilution of $1: 320$, our results could not characterize the variable antigen types (VAT). However, they demonstrated that the bloodstream and the lymph node trypanosome populations isolated simultaneously from the same animal consist of different 'sets of antigenic populations. Seed \& Effron (1973) found a similar antigenic heterogeneity in an agglutination test comparing trypanosome populations simultaneously isolated from the brain and blood of $T$. gambiense-infected Microtus montanus. It may be assumed that the antigenically different populations of bloodstream and lymph node trypanosomes reflect the recently described (Seed, 1978) competition between serologically different clones due to distinct microclimates within various body niches. Syringe passage as well as cyclical transmission lead to a marked VAT heterogeneity as early as the 1st patent parasitaemia (Van Meirvenne, Janssens \& Magnus, 1975; Jenni, 1977; Le Ray, Barry, Easton \& Vickerman, 1977; Le Ray, Barry \& Vickerman, 1978), thus giving a basis for the occurrence of different sets of antigenic populations in different organs of a single animal. Work in progress with clone-specific antisera shows that new VAT can be demonstrated among LF. It remains to be established whether lymph nodes are tissue foci from which a relapse of the parasitaemia can be initiated.

We wish to thank Dr A. M. Jordan, Langford, Bristol, for providing us with pupae material. We thank Miss V. Ghiotto and Miss H. Rieder for their technical assistance and Dr R. Yeates for reading the manuscript. This work was supported in part by the Swiss National Science Foundation, grant no. 3.2360.74.

\section{REFERENCES}

BAKkr, J.R. (1979). Documentation of primary isolates of stocks of Trypanosoma brucei subspecies from Tanzania and Ethiopia. Transactions of the Royal Society of Tropical Medicine and Hygiene 73, 123.

Balber, A. E. (1972). Trypanosoma brucei: fluxes of the morphological variants in intact and X-irradiated mice. Experimental Parasitology 31, 307-19.

BoEHRINGER, S. \& HECKER, H. (1974). Quantitative ultrastructural differences between strains of the Trypanosoma brucei subgroup during transformation in blood. Journal of Protozoology 21, 694-8.

BOEHRINGER, S. \& HECKER, H. (1975). Quantitative ultrastructural investigations of the life cycle of Trypanosoma brucei. A morphometric analysis. Journal of Protozoology 22, 463-7.

Brown, R. C., Evans, D. A. \& Vickerman, K. (1973). Changes in the oxidative metabolism and ultrastructural differentiation of the mitochondrion in Trypanosoma brucei. International Journal for Parasitology 3, 691-704.

BRUN, R. \& JENNI, L. (1977). A new semi-defined medium for Trypanosoma brucei sspp. Acta Tropica 34, 21-33.

Geigy, R., Jenni, L., KaUffmann, M., Onyango, R. J. \& Weiss, N. (1975). Identification of $T$. bruce $i$ subgroup strains isolated from game. Acta Tropica 32, 190-205.

Goodwn, L. G. (1970). The pathology of African trypanosomiasis. Transactions of the Royal Society of Tropical Medicine and Hygiene 64, 797-817.

Hecker, H., BURRI, P. H., Stetger, R. \& Geigy, R. (1972). Morphometric data on the ultrastructure of the pleomorphic bloodforms of Trypanosoma brucei, Plimmer and Bradford, 1899. Acta Tropica 29, 182-98.

HECKER, H., BURRI, P. H. \& BOEHRnNGer, S. (1973). Quantitative ultrastructural differences in the mitochondrion of pleomorphic bloodforms of Trypanosoma brucei. Experientia 29, 901-3.

JENNI, L. (1977). Comparison of antigenic types of Trypanasoma (T.) brucei strains transmitted by Glossina m. morsitans. Acta Tropica 34, 35-41. 

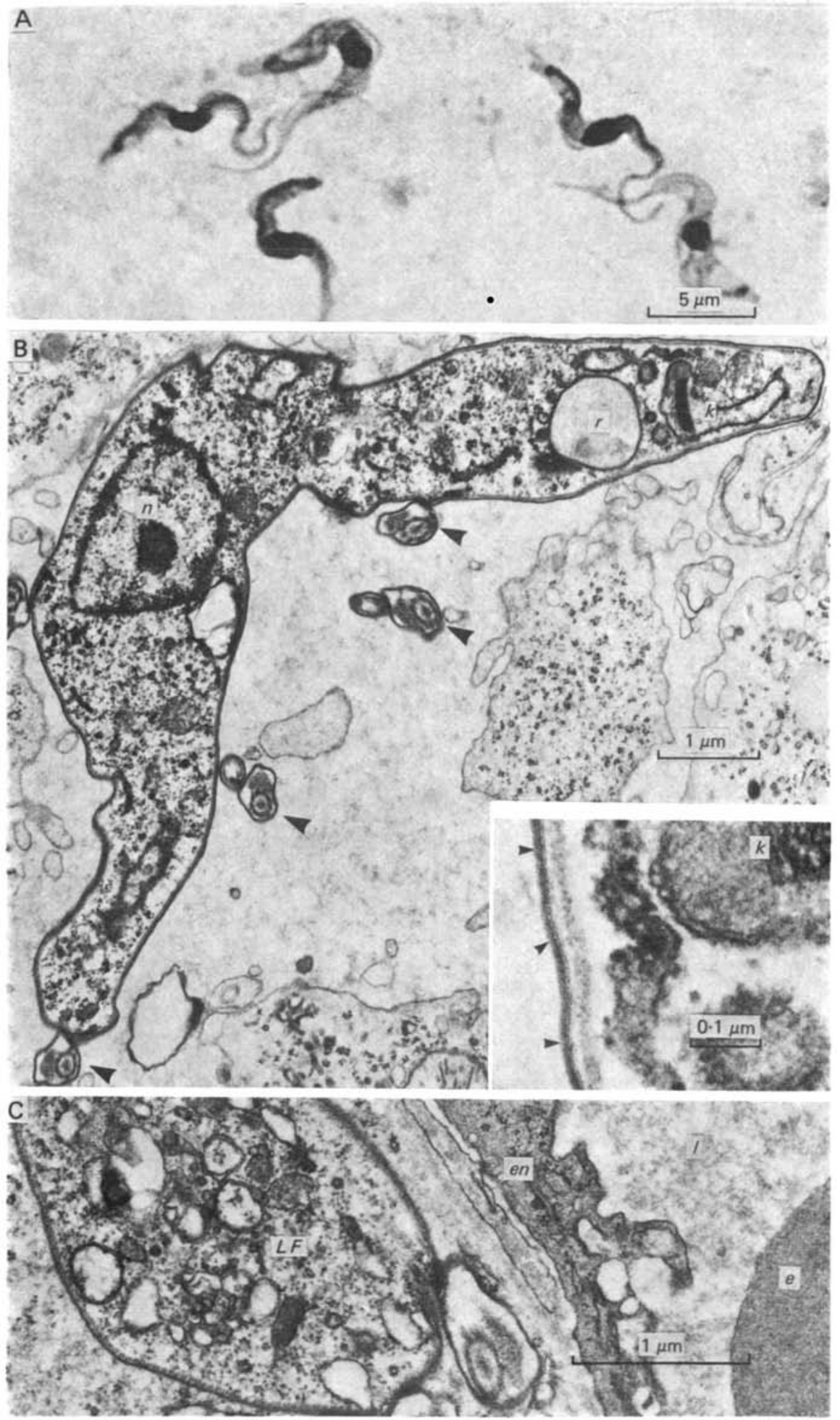

M. TANAER, L. JENAI, H. HECKER AND R. BRUN

(Facing p. 391) 
IE RaY, D., Barry, J. D., Easton, C. \& ViCkerman, K. (1977). First tsetse fly transmission of the 'AnTat' serodeme of Trypanosoma brucei. Annales de la Société belge de Médicine tropicale 57, 369-81.

LE RAY, D., BarRy, J. D. \& VICKERMaN, K. (1978). Antigenic heterogeneity of metacyclic forms of Trypanosoma brucei. Nature, London 273, 300-2.

Losos, G. \& IKEDE, B. O. (1972). Review of pathology of diseases in domestic and laboratory animals caused by Trypanosoma congolense, $T$. vivax, T. brucei, $T$, rhodesiense and $T$. gambiense. Veterinary Pathology 9, 1-71.

NANTULYA, V. M. \& DoYLe, J. J. (1977). Stabilization and preservation of the antigenic specificity of Trypanosoma (Trypanozoon) brucei variant-specific surface antigen by mild fixation techniques. Acta Tropica 34, 313-20.

OrMEROD, W. E. \& Venkatesan, S. (1971). The occult visceral phase of mammalian trypanosomes with special reference to the life cycle of Trypanosoma (Trypanozoon) brucei. Trans. actions of the Royal Society of Tropical Medicine and Hygiene 65, 722-35.

Schuberg, A. \& Boerng, W. (1913). Ueber den Weg der Infektion bei Trypanosomen- und Spirochätenerkrankungen. Deutsche Medizinische Wochenschrift 19, 877-9.

SEED, J. R. (1978). Competition among serologically different clones of Trypanosoma brucei gambiense in vivo. Journal of Protozoology 25, 526-9.

SEED, J. R. \& EFFRON, H. G. (1973). Simultaneous presence of different antigenic populations of Trypanosoma brucei gambiense in Microtus montanus. Parasitology 66, 269-78.

Soltys, M. A., Woo, P. \& GmlICK, A. C. (1969). A preliminary note on the separation and infectivity of tissue forms of Trypanosoma brucei. Transactions of the Royal Society of Tropical Medicine and Hygiene 63, 495-6.

SsenYongA, G. S. Z. \& ADAM, K. M. (1975). The number and morphology of trypanosomes in the blood and lymph of rats infected with Trypanosoma brucei and $T$ congolense. Parsitology 70, 255-61.

Van Meirvenne, N., Janssens, P. G. \& Magnus, E. (1975). Antigenic variation in syringe passaged populations of Trypanosoma (Trypanozoon) brucei. I. Rationalization of the experimental approach. Annales de la Société belge de Médicine tropicale 55, 1-23.

WALKER, P. J. (1970). Techniques for manipulation and maintenance of trypanosome strains in the laboratory. In The African Trypanosomiasis (ed. H. W. Mulligan), pp. 89-96. London: George Allan and Unwin.

\section{EXPLANATION OF PLATE 1}

Trypanosomes isolated from lymph nodes (LF) of Trypanosoma brucei-infected rats.

A. Giemsa-stained smear, note slender-intermediate nature of LF.

B. Longitudinal section of an extravascular LF, kinetoplast $(k)$, reservoir $(r)$, nucleus $(n)$ and flagellum ( $(-)$. Inset: The pellicula of LF is covered with the surface coat (D).

C. Extravascular localization of LF in the lymph node tissue, erythrocyte $(e)$, lumen of the blood vessel $(l)$ and endothelium (en). 